\title{
Distribution and viral load of eight oncogenic types of human papillomavirus (HPV) and HPV 16 integration status in cervical intraepithelial neoplasia and carcinoma
}

\author{
Ming Guo ${ }^{1}$, Nour Sneige ${ }^{1}$, Elvio G Silva ${ }^{1}$, Yee Jee Jan ${ }^{2}$, David E Cogdell ${ }^{1}$, E Lin ${ }^{3}$, \\ Rajyalakshmi Luthra ${ }^{1}$ and Wei Zhang ${ }^{1}$ \\ ${ }^{1}$ Department of Pathology, The University of Texas MD Anderson Cancer Center, Houston, TX, USA; \\ ${ }^{2}$ Department of Pathology, Taichung Veterans General Hospital, Taichung, Taiwan and ${ }^{3}$ Department of \\ Biostatistics and Applied Mathematics, The University of Texas MD Anderson Cancer Center, Houston, \\ TX, USA
}

\begin{abstract}
Current human papillomavirus (HPV) DNA testing using pooled probes, although sensitive, lacks specificity in predicting the risk of high-grade cervical intraepithelial neoplasia (CIN 2/3) progression. To evaluate selected HPV genotyping, viral load, and viral integration status as potential predictive markers for CIN progression, we performed HPV genotyping in formalin-fixed, paraffin-embedded cervical tissue with cervical carcinoma (29 cases) and CINs (CIN 1, 27 cases; CIN 2, 28 cases; CIN 3, 33 cases). General HPVs were screened using consensus primers GP5 +/GP6 + and PGMY09/11. HPV genotyping and viral load measurement were performed using quantitative real-time PCR for eight oncogenic HPV types $(16,18,31,33,35,45,52$, and 58 ). HPV 16 viral integration status was evaluated by measuring HPV 16 E2/E6 ratio. We observed that HPV DNA positivity increased in parallel with the severity of CINs and carcinoma, with $59 \%$ positivity in CIN $1,68 \%$ in CIN $2,76 \%$ in CIN 3, and $97 \%$ in carcinoma $(P$ trend $=0.004)$. The eight oncogenic HPV types were significantly associated with CIN 2/3 (81\%) and carcinoma (93\%) (odds ratio (OR), 15.0; 95\% confidence interval (CI), 5.6739.76; $\boldsymbol{P}<0.0001)$ compared with the unknown HPV types (OR, 2.87; 95\% $\mathrm{Cl}, 0.89-9.22 ; \boldsymbol{P}=0.08)$. HPV 16 was the predominant oncogenic HPV type in CIN $2 / 3(51 \%)$ and carcinoma $(71 \%)$ and integrated significantly more frequently in carcinoma than in CIN 2/3 $(P=0.004)$. No significant differences in viral load were observed across the disease categories. Our findings suggest that selected genotyping for the eight oncogenic HPV types might be useful in separating women with a higher risk of CIN progression from those with a minimal risk. We also conclude that the HPV 16 integration status has potential to be a marker for risk assessment of CIN progression. Modern Pathology (2007) 20, 256-266. doi:10.1038/modpathol.3800737; published online 22 December 2006
\end{abstract}

Keywords: HPV; genotyping; viral load; viral integration; cervical carcinoma; CIN

Human papillomavirus (HPV) is the major risk factor responsible for the development of more than $99 \%$ of cervical cancers and precancerous lesions, that is, cervical intraepithelial neoplasia (CIN 2/3). ${ }^{1-3}$ More than 100 types of HPV have been characterized, with approximately 40 of them being anogenital types. Of these, 15 are associated with cervical carcinoma. ${ }^{4}$ In recent years, HPV DNA testing has emerged as a molecular test that, when

Correspondence: Dr M Guo, MD, Department of Pathology, Unit 85, The University of Texas MD Anderson Cancer Center, 1515 Holcombe Blvd., Houston, TX 77030-4009, USA.

E-mail: mguo@mdanderson.org

Received 10 September 2006; revised 7 November 2006; accepted 9 November 2006; published online 22 December 2006 used in conjunction with cytology, can predict CIN 2/3. ${ }^{5}$ Currently, HPV DNA testing using pooled probe sets, including 13 oncogenic HPV types (Hybrid Capture 2 (HC2), Digene, Gaithersburg, MD, USA) in cytology, can effectively detect almost all of the oncogenic HPV types. Similar to HC2 HPV DNA testing, PCR assays that use consensus primers targeting the highly conserved L1 open reading frame of the HPV genome are also highly sensitive for predicting CIN 2/3, which is essential for cytology specimen triage or primary screening. ${ }^{6,7}$ However, the specificity of HPV DNA testing using pooled or consensus primers to predict CIN $2 / 3$ is low, ${ }^{6}$ with large numbers of women testing positive for HPV DNA, but not having cytologically or histologically confirmed CIN 2/3. 
Recent epidemiological studies have demonstrated that only a few oncogenic HPV types are highly associated with the majority of cervical carcinoma and CIN 2/3.,8 Munoz and Clifford, in separate meta-analyses of pooled data, concluded that eight genotypes of oncogenic HPV $(16,18,31$, $33,35,45,52$, and 58) are closely associated with more than $80 \%$ of cases of cervical carcinoma and CIN 2/3. HPV 16 alone was detected in $54-55 \%$ of cervical carcinomas and $45 \%$ of CIN $2 / 3 . .^{4,8}$ It is unclear whether genotyping for these eight oncogenic HPV types can improve the specificity of HPV testing, which can be used to separate women with clinically relevant diseases that are most likely to progress from those with cervical lesions that mostly regress. Recently published studies have demonstrated that genotyping for HPV 16 and 18 is highly specific in distinguishing women with the greatest risk for CIN 2/3, indicating that genotyping for selected oncogenic HPV types might be a better approach in predicting CIN 2/3 and assessing the risk of precancerous lesion progression. ${ }^{9,10}$ To evaluate the potential utilization of selected HPV genotyping for the risk assessment of precancerous lesion progression, we studied the distribution of the eight oncogenic HPV genotypes in cervical carcinoma and CINs with the hope that it would provide crucial information about the feasibility of using selected genotyping to separate women with the higher risk of lesion progression from those with background risks.

A high HPV 16 viral load has been reported to be associated either with a higher grade of CIN and cervical carcinoma or with a higher risk of developing CIN 2/3 during follow-up, and therefore proposed as a potential marker for risk assessment of CIN progression. ${ }^{11-16}$ Integrating HPV 16 into the host genome of infected cells is considered a critical step for carcinogenesis. ${ }^{17,18}$ HPV 16 integration promotes the transcription of E6 and E7 viral oncogenes. This occurs by disruption or deletion of the viral E1 and/or E2 open reading frame, which releases the suppressive effect of the E2 protein on the viral oncogenes E6 and E7, leading to the activation of E6 and E7 transcription. ${ }^{19-22}$ The E6 and E7 oncogenic potencies of HPV 16 is clearly associated with promoting the transformation and immortalization of infected cells. ${ }^{23}$ Consequently, HPV 16 integration provides a selective growth advantage in the infected cells and is associated with treatment failure or a shortened disease-free survival. $^{20,21,24,25}$ Therefore, the HPV 16 viral integration status may also serve as a useful marker for the risk assessment of cervical precancerous lesion progression. Although studies have suggested that the HPV viral load and integration status might be a marker for cervical precancerous lesion progression, more studies need to be conducted to confirm this. In this study, we used the quantitative real-time-PCR (qRT-PCR) method, which allows for more accurate quantitative analysis of HPV viral load and viral integration with high intra- and inter-assay reproducibilities. $^{26-30}$

\section{Materials and methods}

The Institutional Review Board of The University of Texas MD Anderson Cancer Center approved this study.

\section{Cervical Tissue Specimen Selection}

Archived formalin-fixed paraffin-embedded cervical tissue specimens from 2004 to 2005 were retrieved from the Department of Pathology at the MD Anderson Cancer Center. In consecutive order, 200 cervical tissue specimens were selected from punch biopsies, Loop electrosurgical excision procedures, cone biopsies, and hysterectomies, with pathological diagnoses of normal cervix; CIN 1, 2, or 3; or squamous cell carcinoma. Normal cervical tissue specimens were selected from patients without a history of CIN or abnormal Pap results. Three pathologists independently reviewed the hematoxylin and eosin ( $\mathrm{H}$ and $\mathrm{E}$ )-stained slides. Cases without a consensus in diagnosis were eliminated from the study. Two of the three pathologists reviewed $\mathrm{H}$ and $\mathrm{E}$ slides after block sectioning. Cases were also eliminated from the study for the following reasons: (1) no lesion in the last section of the specimen; (2) insufficient DNA for analysis by spectrophotometry; (3) negative $\beta$-globin amplification by PCR.

\section{Tissue Section for PCR}

The paraffin rolls were cut from each block (10 sections of $10 \mu \mathrm{m}$ thickness) for DNA extraction. The extra sections cut before and after each tissue roll were stained with $\mathrm{H}$ and $\mathrm{E}$ and evaluated to determine the quality of the specimen. To avoid cross contamination, the blade of the microtome was changed for each block.

\section{DNA Extraction}

Formalin-fixed paraffin-embedded tissue rolls were de-paraffinized by xylene $(3 \times)$ followed by an ethanol wash $(3 \times)$. DNA extraction from tissue specimens was performed by using the DNeasy kit (catalog \#69506, Qiagen, Valencia, CA, USA) following the manufacturer's instructions. The extracted DNA was quantified by using a Nanodrop spectrophotometer (Nanodrop Technologies, Wilmington, DE, USA).

\section{HPV DNA Testing Using Consensus Primer-Mediated PCR}

The primers used in the initial screening tests are listed in Table 1. HPV DNA testing and genotyping 
Table 1 Primers for $\beta$-globulin and consensus primers for HPV DNA amplification

\begin{tabular}{|c|c|c|c|c|}
\hline Primers & Sequences $\left(5^{\prime}-3^{\prime}\right)$ & Location & $\mathrm{T}_{m}\left({ }^{\circ} \mathrm{C}\right)$ & References \\
\hline PC04 & CAACTTCATCCACGTTCACC & HBB:nm_000518 & 60.3 & 32 \\
\hline $\mathrm{GH} 20$ & GAAGAGCCAAGGACAGGTAC & HBB:nm_000518 & 63.0 & 32 \\
\hline GP5+ & TTTGTTACTGTGGTAGATACTAC & HPV LI & 51.9 & 33 \\
\hline GP6+ & GAAAAATAAACTGTAAATCATATTC & HPV LI & 53.9 & 33 \\
\hline PGMY11-A & GCACAGGGACATAACAATGG & HPV LI & 62.8 & 34 \\
\hline PGMY11-B & GCGCAGGGCCACAATAATGG & HPV LI & 71.4 & 34 \\
\hline PGMY11-C & GCACAGGGACATAATAATGG & HPV LI & 59.3 & 34 \\
\hline PGMY11-D & GCCCAGGGCCACAACAATGG & HPV LI & 73.8 & 34 \\
\hline PGMY11-E & GCTCAGGGTTTAAACAATGG & HPV LI & 60.9 & 34 \\
\hline PGMY09-F & CGTCCCAAAGGAAACTGATC & HPV LI & 63.3 & 34 \\
\hline PGMY09-G & CGACCTAAAGGAAACTGATC & HPV LI & 57.8 & 34 \\
\hline PGMY09-H & CGTCCAAAAGGAAACTGATC & HPV LI & 61.0 & 34 \\
\hline PGMY09-I & GCCAAGGGGAAACTGATC & HPV LI & 62.1 & 34 \\
\hline PGMY09-J & CGTCCCAAAGGATACTGATC & HPV LI & 60.9 & 34 \\
\hline PGMY09-K & CGTCCAAGGGGATACTGATC & HPV LI & 63.2 & 34 \\
\hline PGMY09-L & CGACCTAAAGGGAATTGATC & HPV LI & 59.9 & 34 \\
\hline PGMY09-M & CGACCTAGTGGAAATTGATC & HPV LI & 58.5 & 34 \\
\hline PGMY09-N & CGACCAAGGGGATATTGATC & HPV LI & 63.0 & 34 \\
\hline PGMY09-P & GCCCAACGGAAACTGATC & HPV LI & 63.1 & 34 \\
\hline PGMY09-Q & CGACCCAAGGGAAACTGGTC & HPV LI & 67.8 & 34 \\
\hline PGMY09-R & CGTCCTAAAGGAAACTGGTC & HPV LI & 60.1 & 34 \\
\hline HMB01 & GCGACCCAATGCAAATTGGT & HPV LI & 68.9 & 34 \\
\hline
\end{tabular}

were carried out randomly without knowledge of the pathological diagnoses. A three-step screening to identify HPV-positive specimens using conventional PCR was performed. First, we performed amplification of $\beta$-globin to determine the quality of the extracted DNA by using primer pc04/gh20 according to Bauer et $a l^{31}$ and AmpliTaq Gold polymerase (Applied Biosystems, Foster City, CA, USA). Briefly, a 25-ng aliquot of genomic DNA was added to a PCR master mixture containing $1 \times$ PCR buffer $(100 \mathrm{mM}$ Tris-HCl, $500 \mathrm{mM} \mathrm{KCl,} \mathrm{pH} \mathrm{8.3),} 200 \mu \mathrm{M}$ each deoxynucleoside triphosphate, $200 \mathrm{nM}$ primer, $1.5 \mathrm{mM} \mathrm{MgCl}_{2}$, and $2.5 \mathrm{U}$ of AmpliTaq Gold DNA polymerase. PCR was performed under the following cycling conditions: $10 \mathrm{~min}$ at $94^{\circ} \mathrm{C}$, followed by $1 \mathrm{~min}$ at $94^{\circ} \mathrm{C}, 1 \mathrm{~min}$ at $40^{\circ} \mathrm{C}, 90 \mathrm{~s}$ at $72^{\circ} \mathrm{C}$, and $5 \mathrm{~min}$ at $72^{\circ} \mathrm{C}$ for 39 cycles.

In the second step of our screening process, specimens positive for $\beta$-globin were screened for HPV by using GP5 +/GP6 + consensus primermediated PCR, which can detect 14 oncogenic HPV genotypes, including the eight major oncogenic HPV types (16, 18, 31, 33, 35, 45, 52, and 58). PCR was performed according to Jacobs et al. ${ }^{32}$ A 25-ng aliquot of genomic DNA was added to the PCR master mixture, which contained $1 \times$ PCR buffer, $200 \mu \mathrm{M}$ each deoxynucleoside triphosphate, $200 \mathrm{nM}$ primer, $3.5 \mathrm{mM} \mathrm{MgCl}_{2}$, and $2.5 \mathrm{U}$ of AmpliTaq Gold DNA polymerase. The cycling conditions were the same as those for $\beta$-globin.

In the third step of screening, specimens that were positive for $\beta$-globin but negative for GP5 +/GP6 + were rescreened for HPV with the second consensus primer sets (PGMY09/11), which also detected all eight major oncogenic HPV genotypes. The PCR assay was performed according to Gravitt et al. ${ }^{33} \mathrm{~A}$ 25-ng aliquot of genomic DNA was used for amplification in a PCR master mixture containing $1 \times$ PCR buffer, $200 \mu \mathrm{M}$ each deoxynucleoside triphosphate, $200 \mathrm{nM}$ primer, $4.0 \mathrm{mM} \mathrm{MgCl}_{2}$, and $7.5 \mathrm{U}$ of AmpliTaq Gold DNA polymerase. PCR was performed under the following cycling conditions: $9 \mathrm{~min}$ at $94^{\circ} \mathrm{C}$, followed by $1 \mathrm{~min}$ at $94^{\circ} \mathrm{C}, 1 \mathrm{~min}$ at $55^{\circ} \mathrm{C}$, and $1 \mathrm{~min}$ at $72^{\circ} \mathrm{C}$ for 39 cycles. The PCR products were visualized with eithiduim bromide staining on a $4 \%$ low-melt agarose gel. Specimens that tested positive for HPV in either of the consensus HPV PCR reactions were subjected to qRT-PCR genotyping.

\section{Quantitative Real-Time PCR Assays for HPV Genotyping}

The eight oncogenic HPV types were amplified by primers and probes targeting the E6/E7 oncogenes of individual HPV genotypes by using qRT-PCR. We used eight corresponding plasmid HPV clones for positive controls. The plasmids containing HPV 16, 18, 35, and 52 were from the American Type Culture Collection (Manassas, VA, USA). HPV 31 was provided by Dr Wayne Lancaster of Wayne State University (MI, USA). HPV 33 was provided by Dr Gerard Orth of the Pasteur Institute, France. HPV 45 was provided by Dr E-M de Villiers of the German Cancer Research Center. HPV 58 was provided by Dr Toshihiko Matsuura of the National Institute of Infectious Diseases in Japan. 
Primers and probes used for real-time PCR are described in Table 2. The Taqman minor grove binder (MGB) probes were used for detecting realtime PCR products. MGB probes were labeled with a corboxyfluorescein reporter dye at the $5^{\prime}$ end and a nonfluorescent quencher at the $3^{\prime}$-end of probe. Primers described by Flores-Munguia et $a{ }^{29}$ for the E6/E7 regions were used for HPV 16, 18, 31, 45, 52, and 58, whereas primers were designed in-house using Primer Express software (Applied Biosystems) for the E6/E7 regions of HPV types 33 and 35. For the HPV 16 integration assay, primers for HPV 16 E2 described by Peitsaro et $a l^{34}$ were used. MGB probes for all assays were designed using Primer Express software, and both probes and primers were purchased from Applied Biosystems.

The specificity of individual HPV primers and probes were validated by using the corresponding plasmid-cloned HPV as previously described..$^{29}$ No cross-reactivity was observed in any of the eight HPV genotypes. High intra- and inter-assay reproducibilities were also obtained (data not shown).

All qRT-PCR studies for HPV genotyping were performed using an ABI PRISM 7900 HT with a 96-well plate (Applied Biosystems). Briefly, $2.5 \mathrm{ng}$ of genomic DNA of each specimen and control, including water as no template control, was added to a $25-\mu \mathrm{l}$ reaction mixture containing $1 \times$ TaqMan Universal PCR Master Mix without AmpErase uracil- $N$-glycosylase and $1 \times$ gene expression assay (Applied Biosystems), $250 \mathrm{nM}$ fluorogenic probe, and $900 \mathrm{nM}$ primer under the following cycling conditions: $10 \mathrm{~min}$ at $95^{\circ} \mathrm{C}$, followed by $15 \mathrm{~s}$ at $95^{\circ} \mathrm{C}$, and $1 \mathrm{~min}$ at $60^{\circ} \mathrm{C}$ for 50 cycles. Each HPV type was assayed on a single 96-well plate, with 40 specimens in duplicate. A standard curve to determine HPV quantity was established, also in duplicate, with a $10 \times$ dilution series ranging from 10 to $1 \times 10^{7}$ copies per well using plasmid-cloned HPV, including water as no template control. A linear relationship was obtained between the log value of the viral copy numbers and the threshold cycle (data not shown). The HPV viral load in the specimen was then determined from standard curve.

The integration status of HPV 16 was determined by measuring the E2/E6 ratio. ${ }^{34}$ The protocol for the E2/E6 assay was modified to include dual standard curves of E2 and E6. All standard curves and

Table 2 Type-specific primers/probes for HPV DNA amplification

\begin{tabular}{|c|c|c|c|c|}
\hline Primer/probes & Sequences $\left(5^{\prime}-3^{\prime}\right)$ & Location & $\mathrm{T}_{m}\left({ }^{\circ} C\right)$ & References \\
\hline Episomal16E2F & AACGAAGTATCCTCTCCTGAAATTATTAG & HPV16E2 & 58.1 & 35 \\
\hline Episomal16E2R & CCAAGGCGACGGCTTTG & HPV16E2 & 59.9 & 35 \\
\hline Episomal16E2P (probe) & 6FAM-CCCCGCCGCGACC-MGB-NFQ & HPV16E2 & 71.9 & a \\
\hline HPV16:520U25 & TTGCAGATCATCAAGAACACGTAGA & HPV16E6-E7 & 59.8 & 30 \\
\hline HPV16:671L24 & CTTGTCCAGCTGGACCATCTATTT & HPV16E6-E7 & 59.5 & 30 \\
\hline HPV16:558U33P (probe) & 6FAM-CATGGAGATACACCTACATTG-MGB-NFQ & HPV16E6-E7 & 69.9 & a \\
\hline HPV18:530U19 & CAACCGAGCACGACAGGAA & HPV18E6-E7 & 59.4 & 30 \\
\hline HPV18:729L21 & CTCGTCGGGCTGGTAAATGTT & HPV18E6-E7 & 60.0 & 30 \\
\hline HPV18:580U37P (probe) & 6FAM-TATGCATGGACCTAAGGCC-MGB-NFQ & HPV18E6-E7 & 73.0 & a \\
\hline HPV31:449F & ATTCCACAACATAGGAGGAAGGTG & HPV31E6-E7 & 59.3 & 30 \\
\hline HPV31:524R & CACTTGGGTTTCAGTACGAGGTCT & HPV31E6-E7 & 59.6 & 30 \\
\hline HPV31:474P (probe) & 6FAM-ACGTTGCATAGCATGTTG-MGB-NFQ & HPV31E6-E7 & 69.9 & a \\
\hline HPV33-CF & AAACCTTTGCAACGATCTGAGGTA & HPV33 E6 & 60.0 & a \\
\hline HPV33-CR & GTTTACATATTCCAAATGGATTTCССTCTCT & HPV33 E6 & 63.4 & a \\
\hline HPV33-CM1 (probe) & 6FAM-ATTTTGCATTTGCAGATTTA-MGB-NFQ & HPV33 E6 & 71.3 & a \\
\hline HPV35-CF & CAAGAATTACAGCGGAGTGAGGTAT & HPV35 E6 & 58.8 & a \\
\hline HPV35-CR & TCCATATGGCTGGCCTTCTCTATA & HPV35 E6 & 59.9 & a \\
\hline HPV35-CM2P (probe) & 6FAM-AATCATAGCATGCAAAGTC-MGB-NFQ & HPV35 E6 & 72.0 & a \\
\hline HPV45:425F & GGACAGTACCGAGGGCAGTGTAA & HPV45 E6-E7 & 61.2 & 30 \\
\hline HPV45:495R & TCCCTACGTCTGCGAAGTCTTTC & HPV45 E6-E7 & 60.7 & 30 \\
\hline HPV45:450TP (probe) & 6FAM-CATGTTGTGACCAGGCAC-MGB-NFQ & HPV45 E6-E7 & 70.9 & a \\
\hline HPV52:78F & GTGCATGAAATAAGGCTGCAGT & HPV52 E6-E7 & 58.0 & 30 \\
\hline HPV52:213R & GTAGGCACATAATACACACGCCA & HPV52 E6-E7 & 58.6 & 30 \\
\hline HPV52:101P(probe) & 6FAM-CAGTGCAAAAAAGAGCTACAA-MGB-NFQ & HPV52 E6-E7 & 69.9 & a \\
\hline HPV58:64F & CCACGGACATTGCATGATTTG & HPV58 E6-E7 & 60.7 & 30 \\
\hline HPV58:144R & CTTTTTGCATTCAACGCATTTCA & HPV58 E6-E7 & 60.9 & 30 \\
\hline HPV58:95TP(probe) & 6FAM-ACATCTGTGCATGAAATC-MGB-NFQ & HPV58 E6-E7 & 71.6 & a \\
\hline
\end{tabular}

${ }^{\mathrm{a}}$ Designed in-house.

$T_{\mathrm{m}}$ is calculated by Primer Express, Applied Biosystems. 
specimens were tested in duplicate, as described above. The E2/E6 ratio was calculated from the same reaction. No E2 amplification was classified as a complete integration. A low E2/E6 ratio represented predominantly integrated forms. Conversely, a high E2/E6 ratio indicated high levels of episomal forms. An E2/E6 ratio that equaled or was greater than one was classified as a complete episomal form.

\section{Statistical Analysis}

Fisher's exact test was used to assess the association between categorical variables. The Cohran-Armitage trend test or the Jonckheere-Terpstra test was used to assess the association between test results and the severity of disease. Wilcoxon's rank sum tests or Kruskal-Wallis tests were used to compare continuous variables between groups. Logistic regression models were used to estimate the odds ratios (ORs) of carcinoma and CIN 2/3, including $95 \%$ confidential intervals (CIs). $P$-values less than 0.05 were considered significant. All computations were carried out at SAS (SAS Institute, Cary, NC, USA).

\section{Results}

The age of women in the study ranged from 18 to 79 years, with a mean age of 40 years and a median age of 41 years. The age distribution was stratified according to pathological diagnoses, as illustrated in Table 3. A total of 137 cases were finally eligible for the study, consisting of 20 cases of normal cervical tissue, 27 cases of CIN 1, 28 cases of CIN 2, 33 cases of CIN 3 , and 29 cases of invasive squamous cell carcinoma.

\section{HPV DNA Detection Using Consensus Primer-Mediated PCR}

All 20 normal cervical specimens tested negative for HPV DNA in either GP5 + /GP6 + or PGMY09/11 consensus primer-mediated PCR assays. Table 4 summarizes HPV DNA positivities in CINs and cervical carcinoma. In GP5 + /GP6 + and PGMY09/

Table 3 Age distribution according to pathological diagnoses

\begin{tabular}{lccc}
\hline Diagnoses & $\begin{array}{c}\text { Number of } \\
\text { cases }\end{array}$ & $\begin{array}{c}\text { Mean of age } \\
\text { (years) }\end{array}$ & $\begin{array}{c}\text { Standard } \\
\text { deviation }\end{array}$ \\
\hline Normal & 20 & 54 & 13 \\
CIN 1 & 27 & 30 & 11 \\
CIN 2 & 28 & 32 & 18 \\
CIN 3 & 33 & 40 & 15 \\
SCC & 29 & 48 & 14 \\
& 137 & &
\end{tabular}

Abbreviations: CIN, cervical intraepithelial neoplasia; SCC, squamous cell carcinoma.
11 primer screenings, $75 \%(88 / 117)$ of all CIN and carcinoma cases were positive for HPV DNA. The percentage of specimens testing positive for HPV DNA increased with the severity of CIN and carcinoma, with 59\% (16/27) in CIN 1, 68\% (19/ $28)$ in CIN 2, 76\% (25/33) in CIN 3, and 97\% (28/29) in carcinoma (Jonckheere-Terpstra trend test, $P=0.0005)$. GP5 + /GP6 + primers detected $94 \%$ (83/88) of all HPV-positive cases. Using PGMY09/ 11 primers, five additional HPV-positive cases were detected from 34 cases negative for GP5 +/GP6 +. The distribution of these five HPV-positive cases was as follows: one (10\%) of 10 cases in CIN 2; two $(20 \%)$ of 10 cases in CIN 3; and two (67\%) of three cases in carcinoma. There was no positive result in the 11 cases of CIN 1. HPV positivity was significantly associated with CIN $2 / 3$ and carcinoma $(P<0.0001)$.

\section{Genotyping of Eight Oncogenic HPV Types Using qRT-PCR}

The distribution of the combined eight oncogenic HPV genotypes, of the coinfection of the eight HPV genotypes, and of the unknown HPV types in cervical carcinoma and CINs are illustrated in Table 5. Of the 88 cases that tested positive for HPV DNA, the genotypes of four cases of CIN 1 and one case of CIN 2 were not further analyzed because of insufficient DNA. In the remaining 83 cases, 67 $(81 \%)$ were positive for at least one of the eight oncogenic HPV types. The presence of the eight oncogenic HPV types increased with the severity of CIN and carcinoma, with 50\% (6/12) in CIN 1, 78\% $(14 / 18)$ in CIN 2, 84\% $(21 / 25)$ in CIN 3, and 93\% $(26 / 28)$ in carcinoma (Jonckheere-Terpstra trend test, $P=0.0001$ ). In contrast, the percentage of unknown HPV types excluding the eight oncogenic HPV types decreased significantly with the severity of CIN and carcinoma, with 50\% (6/12) in CIN 1, $22 \%(4 / 18)$ in CIN 2, $16 \%(4 / 25)$ in CIN 3, and $7 \%$ $(2 / 28)$ in carcinoma (Jonckheere-Terpstra trend test, $P=0.0004)$. Coinfection with two or three HPV

Table 4 HPV positivity in cervical carcinoma and CINs using consensus primer-mediated PCR

\begin{tabular}{lcccc}
\hline Diseases & $\begin{array}{c}\text { Number of } \\
\text { cases }\end{array}$ & \multicolumn{3}{c}{ HPV testing positive } \\
\cline { 3 - 5 } & & $\begin{array}{c}\text { GP5+/GP6+ } \\
(\%)\end{array}$ & PGMY 09/11 & Total (\%) \\
& & $16(59)$ & $0 / 11$ & $16(59)$ \\
CIN 1 & 27 & $18(64)$ & $1 / 10$ & $19(68)$ \\
CIN 2 & 28 & $23(70)$ & $2 / 10$ & $25(76)$ \\
CIN 3 & 33 & $26(90)$ & $2 / 3$ & $28(97)$ \\
Carcinoma & 29 & $83(71)$ & $5 / 34$ & $88(75)$ \\
Total & 117 & &
\end{tabular}

Abbreviations: CIN, cervical intraepithelial neoplasia; HPV, human papilloma virus. 
types was observed in nine cases, of which five were coinfected with HPV 16.

Among the eight oncogenic HPV types, the most frequently detected type was HPV 16, which was detected in $42(51 \%)$ of 83 cases (Table 6). The frequency of HPV 16 positivity increased with the severity of CIN and carcinoma from $39 \%(7 / 18)$ in CIN 2 to $60 \%(15 / 25)$ in CIN 3 and $71 \%(20 / 28)$ in carcinoma. HPV 16 positivity was not observed in any cases of CIN 1. The other seven oncogenic HPV types were found less frequently: HPV 31 and 35, eight (10\%) cases each; HPV 33 and 58, six (7\%) cases each; HPV 52, five (6\%) cases; and HPV 18 and 45 , one ( $1 \%$ ) case each. Only HPV 16 and 18 showed a significantly increased frequency from CIN $2 / 3$ to

Table 5 Distribution of eight oncogenic HPV types, unknown types, and coinfection in cervical carcinoma and CINs

\begin{tabular}{lcccc}
\hline Diseases & $\begin{array}{c}\text { Number of } \\
\text { positive } \\
\text { cases }\end{array}$ & \multicolumn{3}{c}{ HPV genotyping } \\
\cline { 3 - 5 } & & $\begin{array}{c}\text { Eight types } \\
(\%)\end{array}$ & $\begin{array}{c}\text { Unknown } \\
\text { types (\%) }\end{array}$ & $\begin{array}{c}\text { Coinfection } \\
\text { types (\%) }\end{array}$ \\
\hline CIN 1 & 12 & $6(50)$ & $6(50)$ & $31 / 33 / 58$ \\
CIN 2 & 18 & $14(78)$ & $4(22)$ & $16 / 31(2)$, \\
CIN 3 & 25 & $21(84)$ & $4(16)$ & $16 / 52,16 / 31$ \\
Carcinoma & 28 & $26(93)$ & $2(7)$ & $16 / 35,33 / 58$ \\
Total & 83 & $67(81)$ & $16(19)$ & $9(11)$ \\
\hline
\end{tabular}

Abbreviations: CIN, cervical intraepithelial neoplasia; HPV, human papilloma virus. carcinoma compared with the other six oncogenic types and the unknown types of HPV $(P<0.0001)$.

The risk association between HPV types and carcinoma/CIN 2/3 is summarized in Table 7 . The overall HPV positivity was significantly associated with CIN 2/3 and carcinoma (OR, 7.75; 95\% CI, 3.50-17.15; $P<0.0001)$. However, the association between the unknown HPV types and carcinoma/ CIN2/3 was insignificant (OR, 2.87; 95\% CI, 0.899.22; $P=0.08$ ). In contrast, the association became highly significant when comparing the eight oncogenic HPV types with HPV-negative cases (OR, 15.0; 95\% CI, 5.67-39.76; $P<0.0001)$ or when comparing the eight oncogenic HPV types with the combined cases of unknown HPV types positive and HPVnegative cases (OR, 11.5; 95\% CI, 4.57-29.0; $P<0.0001)$. The six types of oncogenic HPV, which did not include HPV 16 and 18, were moderately associated with CIN 2/3 and carcinoma (OR, 3.12; $95 \%$ CI, 1.34-7.25; $P=0.008$ ).

\section{HPV Viral Load and HPV 16 Viral Integration}

The viral load of HPV 16 is illustrated in Figure 1. The median value of the HPV 16 viral load increased with the severity of disease, with ratios of 3.50 in CIN 2, 6.03 in CIN 3, and 6.07 in carcinoma. However, because of the wide range of the viral load for each disease category with broad overlapping, no statistical differences in viral loads were observed in CIN $2 / 3$ and carcinoma $(P=0.15)$. Similarly, the viral loads of the other seven

Table 6 Distribution of the eight oncogenic HPV types in CINs and cervical carcinoma

\begin{tabular}{|c|c|c|c|c|c|c|c|c|c|c|}
\hline \multirow[t]{2}{*}{ Diseases } & \multirow[t]{2}{*}{ Number of positive cases } & \multicolumn{9}{|c|}{ Oncogenic HPV types ${ }^{\mathrm{a}}$} \\
\hline & & $16(\%)$ & 18 & 31 & 33 & 35 & 45 & 52 & 58 & Unknown \\
\hline CIN 1 & 12 & 0 & 0 & 2 & 2 & 0 & 1 & 2 & 1 & 6 \\
\hline CIN 2 & 18 & 7 (39) & 0 & 3 & 3 & 1 & 0 & 1 & 3 & 4 \\
\hline CIN 3 & 25 & $15(60)$ & 0 & 2 & 0 & 4 & 0 & 2 & 0 & 4 \\
\hline SCC & 28 & $20(71)$ & 1 & 1 & 1 & 3 & 0 & 0 & 2 & 2 \\
\hline Total & 83 & $42(51)$ & 1 & 8 & 6 & 8 & 1 & 5 & 6 & 16 \\
\hline
\end{tabular}

Abbreviations: CIN, cervical intraepithelial neoplasia; HPV, human papilloma vorus; SCC, squamous cell carcinoma.

${ }^{\mathrm{a}}$ Including coinfection of the eight oncogenic HPV types.

Table 7 Logistic regression model to estimate the association between HPV infection and cervical carcinoma and CIN2/3

\begin{tabular}{|c|c|c|c|}
\hline HPV types & Comparison & OR $(95 \% C I)$ & $\mathrm{P}$-value \\
\hline Overall HPVs & Positive vs negative & $7.75(3.50-17.15)$ & $<0.0001$ \\
\hline Eight HPV types & Positive vs unknown types/negative & $11.5(4.57-29.0)$ & $<0.0001$ \\
\hline Eight HPV types & Positive vs negative & $15.0(5.67-39.76)$ & $<0.0001$ \\
\hline Six HPV types excluding HPV16, 18 & Positive vs negative & $3.12(1.34-7.25)$ & 0.008 \\
\hline Unknown HPV types & Positive vs negative & $2.87(0.89-9.22)$ & 0.08 \\
\hline
\end{tabular}

Abbreviations: CIN, cervical intraepithelial neoplasia; CI, confidence interval; HPV, human papilloma virus; OR, odds ratio. 


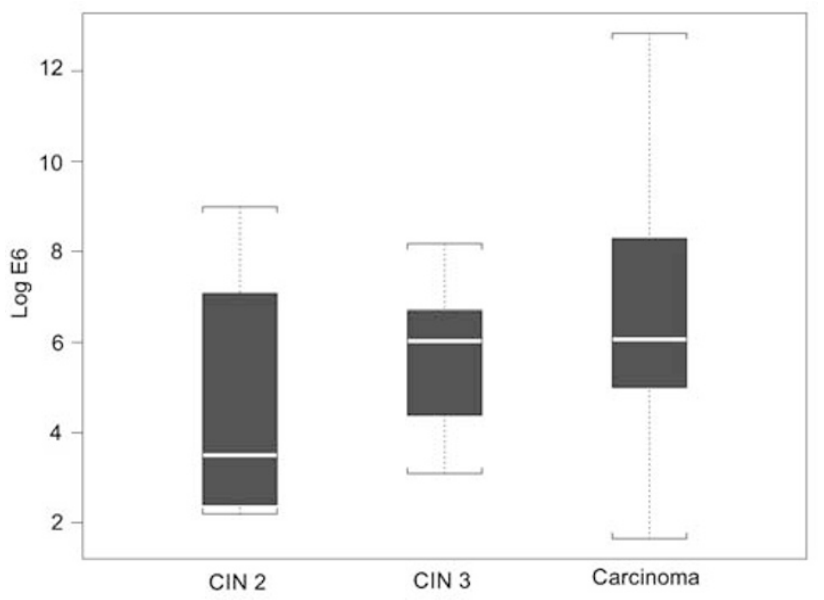

Figure 1 HPV 16 viral load in CIN 2/3 and cervical carcinoma $(P=0.15)$.

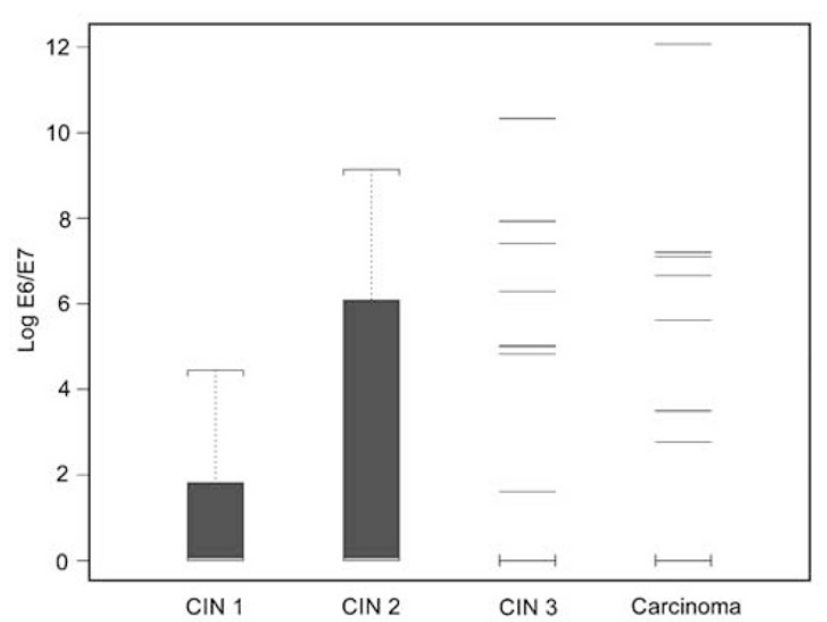

Figure 2 Combined HPV viral load of seven oncogenic HPV types, excluding of HPV 16, in CINs and cervical carcinoma $(P=0.74)$.

genotyped HPV types, excluding HPV 16, also increased with the severity of disease. However, no significant differences in the viral load were observed across the disease categories $(P=0.74)$ (Figure 2).

The HPV 16 E2/E6 ratio was tested in a total of 42 cases. The median E2/E6 ratio was significantly lower in carcinoma (0.13) than in CIN 2 (0.46) and CIN $3(0.50)(P=0.004)$, indicating a significantly higher integrated form of HPV 16 in cervical carcinoma than in CIN 2/3 (Figure 3). Using an E2/ E6 ratio of 0.6 as a cutoff, all of the carcinoma cases $(20 / 20)$ and $64 \%(14 / 22)$ of the CIN $2 / 3$ cases were included below the range of 0.6 (Figure 4). Based on the E2/E6 ratio, mixed episomal and integrated forms of HPV 16 were most frequently observed in CIN 2/3 (68\%, 15/22) and carcinoma (65\%, 13/20). Three cases containing predominantly episomal

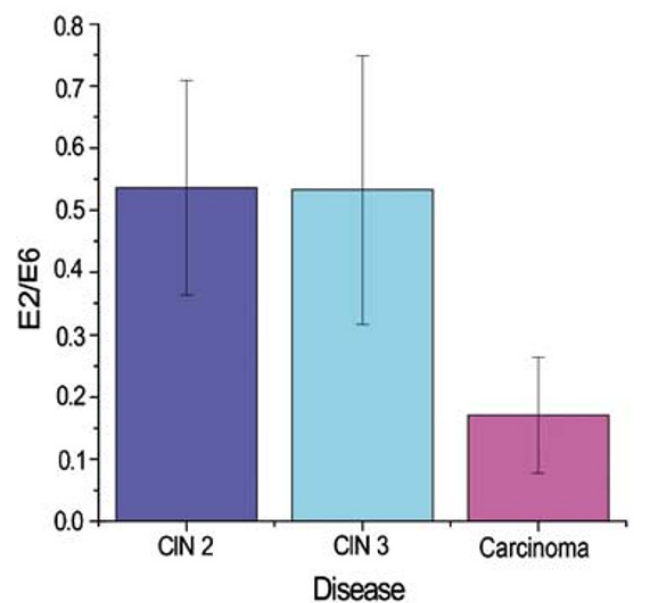

Figure 3 HPV 16 viral integration status (E2/E6 ratio) in CIN 2/3 and cervical carcinoma.

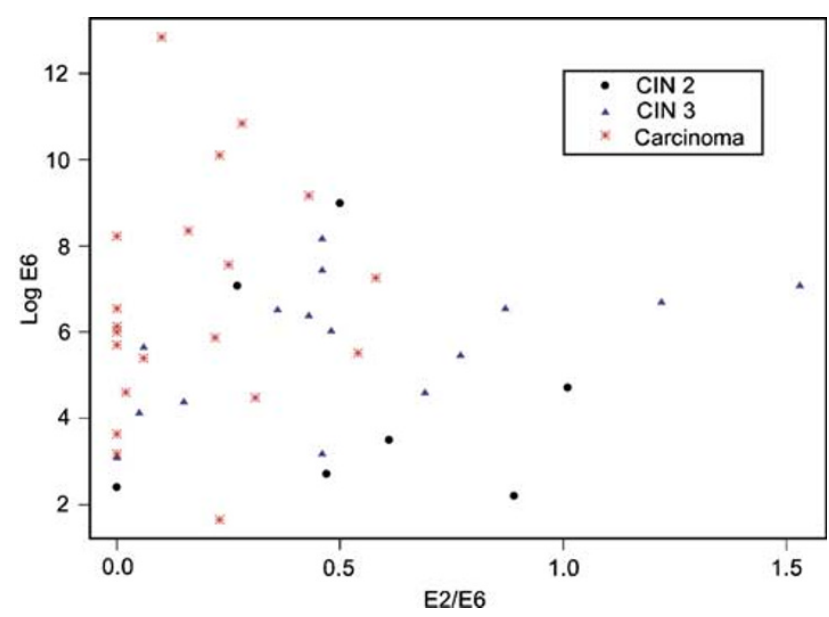

Figure 4 Distribution of HPV 16 viral load and integration status in CIN 2/3 and cervical carcinoma.

Table 8 HPV16 physical status in cervical carcinoma and CIN 2/3

\begin{tabular}{lcc}
\hline Physical status & CIN 2/3 cases (\%) & $\begin{array}{c}\text { Carcinoma cases } \\
(\%)\end{array}$ \\
\hline Episomal & $3(14)$ & 0 \\
Integrated & $4(18)$ & $7(35)$ \\
Mixed & $15(68)$ & $13(65)$ \\
Total & 22 & 20 \\
\hline
\end{tabular}

Abbreviation: CIN, cervical intraepithelial neoplasia.

form of HPV 16 were observed in CIN 2/3 (14\%, $3 / 22$ ), whereas the cases with predominantly integrated form were observed more frequently in carcinoma $(35 \%, 7 / 20)$ compared with CIN $2 / 3$ $(18 \%, 4 / 22)$ (Table 8). 


\section{Discussion}

In this cross-sectional study, we demonstrated a significantly close association between the eight oncogenic HPV types and cervical carcinoma and CIN 2/3, compared with the unknown HPV types. HPV 16, the predominant HPV genotype with a significant association with cervical carcinoma and CIN 2/3, integrated significantly more frequently in carcinoma than in CIN 2/3. The viral load of the eight oncogenic HPV types increased with the severity of the disease, but without significant differences between CIN and carcinoma.

Our HPV DNA testing with GP5 +/GP6 + consensus primers demonstrated a high HPV positivity, which is consistent with the published pooled data. ${ }^{4}$ Using the second consensus primer sets (PGMY09/ 11) to screen specimens that were negative for GP5 +/GP6 + further increased the HPV positivity. Although both GP5 + /GP6 + and PGMY09/11 primers broadly cover most of the oncogenic HPV types, each primer set has a certain complementary coverage. ${ }^{35}$ That is, compared with GP5 + /GP6+, PGMY09/11 is more sensitive in detecting multiple HPV infections as well as HPV 52 infection. ${ }^{35}$ In our study of 88 HPV-positive cases, 83 (94\%) were detected using GP5 + /GP6 + primer sets, indicating that $6 \%$ of HPV-positive cases would have been missed if only GP5 + /GP6 + primers had been used and most of these potentially missed HPV-positive cases were CIN 3 and carcinoma (4/5).

We demonstrated that the risk associated with CIN $2 / 3$ and carcinoma was predominantly determined by the combined eight oncogenic HPV types compared with the unknown HPV types (Figure 5). Our data further showed that out of the eight oncogenic HPV types, HPV 16 and 18 were most significantly associated with CIN 3 and cervical carcinoma

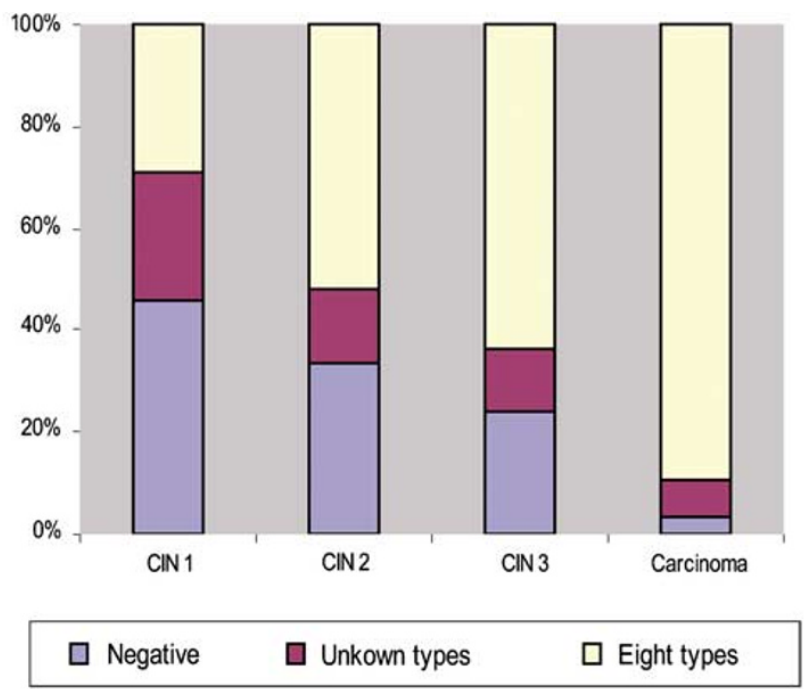

Figure 5 Distribution of HPVs and the eight oncogenic types in CINs and cervical carcinoma.
(Figure 6). The six other oncogenic HPV genotypes were also associated with CIN $2 / 3$ and carcinoma but to a lesser extent. This observation is in keeping with recently published prospective studies that demonstrated that HPV 16 and 18 were the most clinically relevant HPV genotypes to be significantly associated with precancerous lesion progression compared with other oncogenic HPV genotypes which only showed background risk for developing CIN 2/3 lesions. ${ }^{9,10,36}$ Our findings suggest that HPV genotyping for the eight most clinically relevant oncogenic HPV types might be more specific and cost-effective than genotyping for most of the anogenital HPV types to identify women with higher risk of developing CIN 2/3 or cervical carcinoma.

In our study, we observed geographic variability of oncogenic HPV distribution in cervical carcinoma and CIN 2/3. We found that the distribution patterns of the individual HPV types differed from those shown by a pooled data from North American population and by our previous study in Mississippi, which showed HPV 16 to be the most prevalent, followed by HPV 18 and 31. ${ }^{8,37}$ In our current study, HPV 16 was the most prevalent type, followed by HPV 31 and HPV 35. HPV 18 was under-represented in our carcinoma and CIN 2/3 groups. However, this could be due to the fact that in our study, only squamous cell carcinoma was selected for HPV genotyping. Furthermore, HPV 18, which is more commonly associated with endocervical adenocarcinoma, has been reported to be difficult to detect in cytology specimens. ${ }^{38}$ Schiffman et $a l^{36}$ also reported that carcinoma cases positive for HPV 18 were missed during the baseline screening. Owing to our small sample size, a study with a large sample size or pooled data from the same geographic area may help to clarify the issue.

HPV 16 type-specific persistent infection has been attributed partially to its oncogenic potency and proposed to be used as a reliable marker indepen-

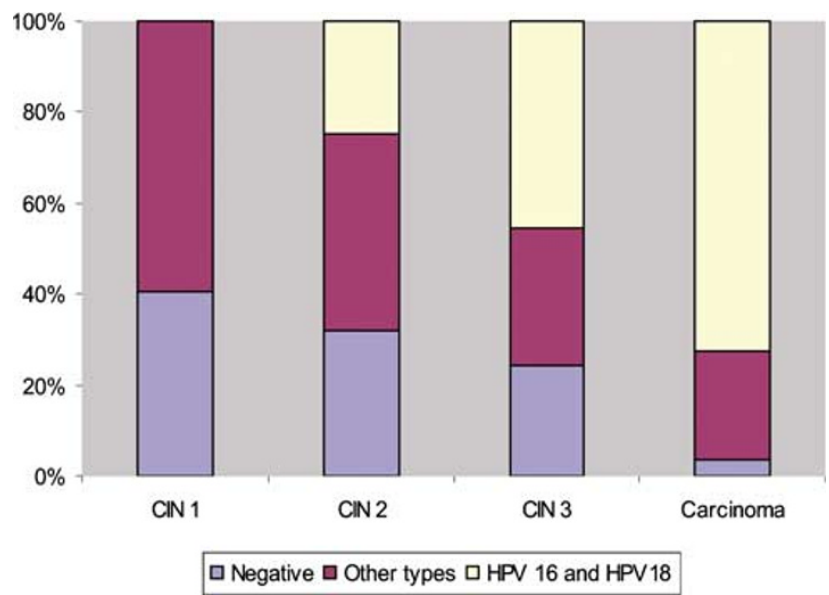

Figure 6 Distribution of HPVs and HPV 16 and HPV 18 in CINs and cervical carcinoma. 
dent of morphology for cervical precancerous lesion progression. ${ }^{36,39,40}$ Although the mechanism that causes persistent infection of HPV 16 is not completely clear, it has been reported to be associated with viral integration into the human genome. ${ }^{41,42}$ In contrast, the oncogenic HPV types, such as HPV 52 and 58, was reported significantly less likely to be integrated into the cells of cervical carcinoma. ${ }^{43}$ We demonstrated the integrated form of HPV 16 in all of the carcinoma specimens and significantly increased HPV 16 integration in cervical carcinoma compared with CIN 2/3, supporting the notion that HPV 16 integration into the host genome is an important step of carcinogenesis. ${ }^{17,18}$ In most published studies of cervical carcinoma, the integrated forms of HPV 16 were also observed in either all or a majority of the specimens. ${ }^{42,43}$ AriasPulido et $a l^{22}$ recently conducted a study of formalin-fixed paraffin-embedded as well as fresh cervical specimens using qRT-PCR and reported a relatively low proportion of the integrated type of HPV 16 in carcinoma in situ $(30.3 \%)$ and in invasive carcinoma $(60.9 \%)$. The discrepancies of HPV 16 integration in the published studies may be resulted by different primers targeting different E1 or E2 regions and the effect of the adjacent tissue around the targeted lesion. ${ }^{22}$ The evaluation of HPV 16 integration using qRT-PCR can also be affected by the quantity of the episomal form of the virus in specimens. ${ }^{22}$ Nevertheless, Given the fact that the mixed integrated and episomal forms of HPV 16 are the predominant pattern in CINs or even normal cervix, ${ }^{30,34,42,44,45}$ the relative ratio of E2/E6, instead of the prevalence of completely integrated HPV 16, may be more specific in predicting CIN progression and may have potential as a predictive marker. However, our small sample size in a cross-sectional study precludes any conclusion about the use of the HPV 16 E2/E6 ratio cutoff to predict CIN progression. Longitudinal studies with large sample sizes are required to determine the significance of the HPV 16 E2/E6 ratio as a predictive marker for CIN $2 / 3$ and carcinoma.

In previous studies, a high viral load of HPV 16 was reported to be associated with persistent infection and the development of CIN $2 / 3$ and cervical carcinoma ${ }^{11,12,16,30,46}$ and might have predictive value for CIN 2/3 or carcinoma. ${ }^{14,42,47,48}$ Most studies using qRT-PCR assays in cytology specimens have demonstrated an increased HPV 16 viral load in higher-grade squamous intraepithelial lesions. However, a controversial observation has been reported, showing inconsistent results with significantly less HPV 16 viral load in high-grade squamous intraepithelial lesions than low-grade squamous intraepithelial lesions. ${ }^{49}$ Our results in formalin-fixed paraffin-embedded material showed that the HPV 16 viral load was proportionally associated with the grade of cervical lesions, with higher viral loads in higher grades of the lesions, but lack of statistical significance.
Our study is limited by a lack of specimen homogeneity, which can be achieved by microdissection. Viral load evaluation can be affected by the heterogeneity of specimens, such as the extent of CIN 1 surrounding CIN 3 in histology specimens as well as the numbers of abnormal cells in cytology specimens. ${ }^{50}$ The specimen heterogeneity in our study may partially explain the broad overlapping of the HPV 16 viral load range in CIN $2 / 3$ and carcinoma. Similar observations have also been made in cytology specimens, which makes it difficult to define a cutoff value for risk prediction. $^{30,51}$ Our cross-sectional data also limit the evaluation of kinetics of the HPV 16 viral load, which may be more valuable in predicting CIN $2 / 3$ and cervical carcinoma, as demonstrated in longitudinal studies that a higher viral load is highly predictive for precancerous lesion progression. ${ }^{12,15}$ Periodic evaluation of the HPV viral load may be useful in monitoring viral clearing and predicting cervical precancerous lesion progression, but requires more studies to determine the value of HPV viral load as a predictive marker. ${ }^{52,53}$

\section{Acknowledgements}

We thank the following scientists for kindly providing the plasmid cloned HPV DNA: Dr Wayne Lancaster, Wayne State University, MI, USA (HPV 31); Dr Gerard Orth, Pasteur Institute, France (HPV 33); Dr E-M de Villiers, German Cancer Research Center, Germany (HPV 45); Dr Toshihiko Matsuura, National Institute of Infectious Diseases, Japan (HPV 58). We thank Alen Silverman, Applied Biosystems, for designing the probes and part of the primers for HPV genotyping using real-time PCR and Valerie Dunmire, Erik E Guerra, MD Anderson Cancer Center, for technical support. We also thank Mrs Martha Morrison, Scientific Publications, MD Anderson Cancer Center, for editing the manuscript.

\section{References}

1 Bosch FX, Manos MM, Munoz N, et al. Prevalence of human papillomavirus in cervical cancer: a worldwide perspective. International biological study on cervical cancer (IBSCC) Study Group. J Natl Cancer Inst 1995;87:796-802.

2 Walboomers JM, Jacobs MV, Manos MM, et al. Human papillomavirus is a necessary cause of invasive cervical cancer worldwide. J Pathol 1999;189:12-19.

3 zur Hausen H. Papillomaviruses and cancer: from basic studies to clinical application. Nat Rev Cancer 2002;2:342-350.

4 Munoz N, Bosch FX, de Sanjose S, et al. Epidemiologic classification of human papillomavirus types associated with cervical cancer. N Engl J Med 2003; 348:518-527.

5 Wright Jr TC, Cox JT, Massad LS, et al. 2001 consensus guidelines for the management of women with cervical 
intraepithelial neoplasia. Am J Obstet Gynecol 2003; 189:295-304.

6 Kulmala SM, Syrjanen S, Shabalova I, et al. Human papillomavirus testing with the hybrid capture 2 assay and PCR as screening tools. J Clin Microbiol 2004; 42:2470-2475.

7 Schiffman M, Wheeler CM, Dasgupta A, et al. A comparison of a prototype PCR assay and hybrid capture 2 for detection of carcinogenic human papillomavirus DNA in women with equivocal or mildly abnormal papanicolaou smears. Am J Clin Pathol 2005;124:722-732.

8 Clifford GM, Smith JS, Aguado T, et al. Comparison of HPV type distribution in high-grade cervical lesions and cervical cancer: a meta-analysis. $\mathrm{Br} \mathrm{J}$ Cancer 2003;89:101-105.

9 Castle PE, Solomon D, Schiffman M, et al. Human papillomavirus type 16 infections and 2-year absolute risk of cervical precancer in women with equivocal or mild cytologic abnormalities. J Natl Cancer Inst 2005;97:1066-1071.

10 Khan MJ, Castle PE, Lorincz AT, et al. The elevated 10year risk of cervical precancer and cancer in women with human papillomavirus (HPV) type 16 or 18 and the possible utility of type-specific HPV testing in clinical practice. J Natl Cancer Inst 2005;97: 1072-1079.

11 Josefsson AM, Magnusson PK, Ylitalo N, et al. Viral load of human papilloma virus 16 as a determinant for development of cervical carcinoma in situ: a nested case-control study. Lancet 2000;355:2189-2193.

12 Ylitalo N, Sorensen P, Josefsson AM, et al. Consistent high viral load of human papillomavirus 16 and risk of cervical carcinoma in situ: a nested case-control study. Lancet 2000;355:2194-2198.

13 Dalstein V, Riethmuller D, Pretet JL, et al. Persistence and load of high-risk HPV are predictors for development of high-grade cervical lesions: a longitudinal French cohort study. Int J Cancer 2003;106:396-403.

14 Lo KW, Yeung SW, Cheung TH, et al. Quantitative analysis of human papillomavirus type 16 in cervical neoplasm: a study in Chinese population. J Clin Virol 2005;34:76-80.

15 Monnier-Benoit S, Dalstein V, Riethmuller D, et al. Dynamics of HPV16 DNA load reflect the natural history of cervical HPV-associated lesions. J Clin Virol 2006;35:270-277.

16 Moberg M, Gustavsson I, Wilander E, et al. High viral loads of human papillomavirus predict risk of invasive cervical carcinoma. Br J Cancer 2005;92:891-894.

17 Pett MR, Alazawi WO, Roberts I, et al. Acquisition of high-level chromosomal instability is associated with integration of human papillomavirus type 16 in cervical keratinocytes. Cancer Res 2004;64:1359-1368.

18 Pett MR, Herdman MT, Palmer RD, et al. Selection of cervical keratinocytes containing integrated HPV16 associates with episome loss and an endogenous antiviral response. Proc Natl Acad Sci USA 2006; 103:3822-3827.

19 Romanczuk H, Howley PM. Disruption of either the E1 or the E2 regulatory gene of human papillomavirus type 16 increases viral immortalization capacity. Proc Natl Acad Sci USA 1992;89:3159-3163.

20 Jeon S, Allen-Hoffmann BL, Lambert PF. Integration of human papillomavirus type 16 into the human genome correlates with a selective growth advantage of cells. J Virol 1995;69:2989-2997.
21 Kalantari M, Karlsen F, Kristensen G, et al. Disruption of the E1 and E2 reading frames of HPV 16 in cervical carcinoma is associated with poor prognosis. Int J Gynecol Pathol 1998;17:146-153.

22 Arias-Pulido H, Peyton CL, Joste NE, et al. Human papillomavirus type 16 integration in cervical carcinoma in situ and in invasive cervical cancer. J Clin Microbiol 2006;44:1755-1762.

23 Munger K, Howley PM. Human papillomavirus immortalization and transformation functions. Virus Res 2002;89:213-228.

24 Unger ER, Vernon SD, Thoms WW, et al. Human papillomavirus and disease-free survival in FIGO stage Ib cervical cancer. J Infect Dis 1995;172:1184-1190.

25 Vernon SD, Unger ER, Miller DL, et al. Association of human papillomavirus type 16 integration in the E2 gene with poor disease-free survival from cervical cancer. Int J Cancer 1997;74:50-56.

26 Moberg M, Gustavsson I, Gyllensten U. Real-time PCRbased system for simultaneous quantification of human papillomavirus types associated with high risk of cervical cancer. J Clin Microbiol 2003;41:3221-3228.

27 Gravitt PE, Peyton C, Wheeler C, et al. Reproducibility of HPV 16 and HPV 18 viral load quantitation using TaqMan real-time PCR assays. J Virol Methods 2003;112:23-33.

28 Lefevre J, Hankins C, Pourreaux K, et al. Real-time PCR assays using internal controls for quantitation of HPV16 and beta-globin DNA in cervicovaginal lavages. J Virol Methods 2003;114:135-144.

29 Flores-Munguia R, Siegel E, Klimecki WT, et al. Performance assessment of eight high-throughput PCR assays for viral load quantitation of oncogenic HPV types. J Mol Diagn 2004;6:115-124.

30 Fontaine J, Hankins C, Mayrand MH, et al. High levels of HPV-16 DNA are associated with high-grade cervical lesions in women at risk or infected with HIV. Aids 2005;19:785-794.

31 Bauer HM, Ting Y, Greer CE, et al. Genital human papillomavirus infection in female university students as determined by a PCR-based method. JAMA 1991;265:472-477.

32 Jacobs MV, Snijders PJ, van den Brule AJ, et al. A general primer GP5+/GP6(+)-mediated PCR-enzyme immunoassay method for rapid detection of 14 highrisk and 6 low-risk human papillomavirus genotypes in cervical scrapings. J Clin Microbiol 1997;35: 791-795.

33 Gravitt PE, Peyton CL, Alessi TQ, et al. Improved amplification of genital human papillomaviruses. J Clin Microbiol 2000;38:357-361.

34 Peitsaro P, Johansson B, Syrjanen S. Integrated human papillomavirus type 16 is frequently found in cervical cancer precursors as demonstrated by a novel quantitative real-time PCR technique. J Clin Microbiol 2002;40:886-891.

35 Chan PK, Cheung TH, Tam AO, et al. Biases in human papillomavirus genotype prevalence assessment associated with commonly used consensus primers. Int J Cancer 2006;118:243-245.

36 Schiffman M, Herrero $\mathrm{R}$, Desalle $\mathrm{R}$, et al. The carcinogenicity of human papillomavirus types reflects viral evolution. Virology 2005;337:76-84.

$37 \mathrm{Hu} \mathrm{L}$, Guo M, He Z, et al. Human papillomavirus genotyping and p16INK4a expression in cervical intraepithelial neoplasia of adolescents. Mod Pathol 2005;18:267-273. 
38 Andersson S, Rylander E, Larsson B, et al. The role of human papillomavirus in cervical adenocarcinoma carcinogenesis. Eur J Cancer 2001;37:246-250.

39 Kjaer SK, van den Brule AJ, Paull G, et al. Type specific persistence of high risk human papillomavirus (HPV) as indicator of high grade cervical squamous intraepithelial lesions in young women: population based prospective follow up study. BMJ 2002;325:572.

40 Trimble CL, Piantadosi S, Gravitt P, et al. Spontaneous regression of high-grade cervical dysplasia: effects of human papillomavirus type and HLA phenotype. Clin Cancer Res 2005;11:4717-4723.

41 Brummer O, Hollwitz B, Bohmer G, et al. Human papillomavirus-type persistence patterns predict the clinical outcome of cervical intraepithelial neoplasia. Gynecol Oncol 2006;102:517-522.

42 Kulmala SM, Syrjanen SM, Gyllensten UB, et al. Early integration of high copy HPV16 detectable in women with normal and low grade cervical cytology and histology. J Clin Pathol 2006;59:513-517.

43 Ho CM, Chien TY, Huang SH, et al. Integrated human papillomavirus types 52 and 58 are infrequently found in cervical cancer, and high viral loads predict risk of cervical cancer. Gynecol Oncol 2006; 102:54-60.

44 Ueda Y, Enomoto T, Miyatake T, et al. Monoclonal expansion with integration of high-risk type human papillomaviruses is an initial step for cervical carcinogenesis: association of clonal status and human papillomavirus infection with clinical outcome in cervical intraepithelial neoplasia. Lab Invest 2003;83: 1517-1527.

45 Andersson S, Safari H, Mints M, et al. Type distribution, viral load and integration status of high-risk human papillomaviruses in pre-stages of cervical cancer (CIN). Br J Cancer 2005;92:2195-2200.
46 Moberg M, Gustavsson I, Gyllensten U. Type-specific associations of human papillomavirus load with risk of developing cervical carcinoma in situ. Int J Cancer 2004;112:854-859.

47 Lefevre J, Hankins C, Money D, et al. Human papillomavirus type 16 viral load is higher in human immunodeficiency virus-seropositive women with high-grade squamous intraepithelial lesions than in those with normal cytology smears. J Clin Microbiol 2004;42:2212-2215.

48 van Duin M, Snijders PJ, Schrijnemakers HF, et al. Human papillomavirus 16 load in normal and abnormal cervical scrapes: an indicator of CIN II/III and viral clearance. Int J Cancer 2002;98:590-595.

49 Flores R, Papenfuss M, Klimecki WT, et al. Crosssectional analysis of oncogenic HPV viral load and cervical intraepithelial neoplasia. Int $\mathrm{J}$ Cancer 2006;118:1187-1193.

50 Sherman ME, Wang SS, Wheeler CM, et al. Determinants of human papillomavirus load among women with histological cervical intraepithelial neoplasia 3: dominant impact of surrounding low-grade lesions. Cancer Epidemiol Biomarkers Prev 2003;12:1038-1044.

51 Swan DC, Tucker RA, Tortolero-Luna G, et al. Human papillomavirus (HPV) DNA copy number is dependent on grade of cervical disease and HPV type. J Clin Microbiol 1999;37:1030-1034.

52 Snijders PJ, van den Brule AJ, Meijer CJ. The clinical relevance of human papillomavirus testing: relationship between analytical and clinical sensitivity. J Pathol 2003;201:1-6.

53 Snijders PJ, Hogewoning CJ, Hesselink AT, et al. Determination of viral load thresholds in cervical scrapings to rule out CIN 3 in HPV 16, 18, 31 and 33-positive women with normal cytology. Int J Cancer 2006;119:1102-1107. 